\title{
Coulisses
}

Revue de théâtre

\section{Le pacte et le legs : Les réécritures de Faust dans Moby Dick d'Herman Melville}

Anne Bourse

\section{(2) OpenEdition}

1 Journals

Édition électronique

URL : https://journals.openedition.org/coulisses/384

DOI : 10.4000/coulisses.384

ISSN : 2546-9460

Éditeur

Presses universitaires de Franche-Comté

\section{Édition imprimée}

Date de publication : 31 décembre 2011

Pagination : 127-136

ISBN : 978-2-84867-404-9

ISSN : $1150-594 X$

\section{Référence électronique}

Anne Bourse, «Le pacte et le legs : Les réécritures de Faust dans Moby Dick d'Herman Melville», Coulisses [En ligne], 43 | Automne 2011, mis en ligne le 30 novembre 2016, consulté le 29 décembre 2022. URL : http://journals.openedition.org/coulisses/384 ; DOI : https://doi.org/10.4000/coulisses 384 


\title{
Le pacte et le legs : Les réécritures de Faust dans Moby Dick d'Herman Melville
}

\author{
Anne Bourse
}

1 Ainsi qu'en témoignent les quatre-vingt épigraphes postées par Melville en exergue de Moby Dick, offrant en guise de préambule une recension des considérations cétologiques amassées depuis la Bible jusqu'aux chansons populaires de Nantucket, l'auteur du roman-monstre a lui-même vécu comme un personnage faustien. Lecteur avide débordant littéralement de citations, de références et de tutelles prestigieuses, il est l'alchimiste "saugrenu» qui s'est efforcé de transformer la graisse de baleine ("blubber») en un immense in-folio, en parchemin hiéroglyphique (sur)chargé contre vents et marées, roulis et tangage, d'énoncer la fuyante Vérité et de contribuer, au même titre que The Prairie de Cooper, The Narrative of Arthur Gordon Pym de Poe et The Scarlet Letter de Hawthorne, à la glorieuse « Renaissance » des lettres américaines ${ }^{1}$. C'est une perspective éminemment faustienne que celle adoptée par Melville en 1851: comment sortir du temple babélien de la connaissance livresque et que faire, concrètement, poétiquement, des monceaux de savoir arrachés à la patience de l'étude et stockés en fond de cale de la création littéraire ? Les fondre dans les cuves brûlantes du Pequod afin d'en extraire l'huile susceptible d'éclairer les pistes encore inexplorées $\mathrm{du}$ Pacifique ? Les laisser pourrir et redevenir poussière à l'instar du " pauvre diable de ver de terre" («grubworm of a poor devil») qui hante de son érudition tuberculeuse l'avant-texte de Moby Dick? Les saborder énergiquement afin de révéler la page blanche originaire («blank») sur laquelle signer de son nom propre? Les abandonner au large comme on le ferait de bouteilles à la mer, avant de s'élancer hardiment, enfin délesté $\mathrm{du}$ " bric-à-brac ancestral » ("Urväter-Hausrat drein gestopft »), au-delà de tout cadastre et de toute frontière connue?

2 Entre le corpus mouvant qu'alimentent les multiples versions de Faust et la chair gargantuesque du roman-monstre surgit ainsi l'épineuse question de l'héritage à consommer et de l'autorité à conquérir. Comparable à l'écrasant patrimoine shakespearien dont Melville revendique avec détermination la labilité et l'accessibilité pour ses 
successeurs anglophones ${ }^{3}$, la réécriture de Faust par Moby Dick cristallise de manière exemplaire le problème de l'indépendance des écrivains américains à l'égard de la tradition littéraire et philosophique des siècles passés; conflit de perspective et de regard que résume Emerson dès l'introduction de Nature (1836) :

Our age is retrospective. It builds the sepulchres of the fathers. It writes biographies, histories, and criticism. The foregoing generations beheld God and nature face to face; we, through their eyes. Why should not we also enjoy an original relation to the universe? [...] why should we grope among the dry bones of the past, or put the living generation into masquerade out of its faded wardrobe?

The sun shines to-day also ${ }^{4}$.

Cette menaçante emprise du legs qui obstrue la perception émerveillée du Nouveau Monde, cette " hantise jamais exorcisée de l'hypothèse coloniale » remarquablement analysée par Pierre-Yves Pétillon dans La Grand-route ${ }^{5}$, dont les œuvres inquiètes de Hawthorne et de Faulkner sont d'autres frappantes illustrations, se précise dans le rapport qu'entretient Melville avec Marlowe et Goethe. Au-delà de l'émancipation culturelle, littéraire et linguistique des auteurs d'Amérique du Nord vis-à-vis de la Grande-Bretagne, Moby Dick interroge la liquidation de l'héritage plus largement européen dont Faust est à la fois le parangon et, comme nous le rappelle justement le Méphistophélès de Valéry ${ }^{6}$, la très matérielle récapitulation.

L'ingestion de la cartographie faustienne et de la figure palimpseste de Faust au sein de la «baleine de livre" relève donc de la généalogie, au sens que Nietzsche et Foucault accordent à ce terme : nourri d'érudition et de patientes relectures, c'est un rapport critique au passé, qui scrute "le corps tout imprimé d'histoire, l'histoire ruinant le corps $»^{7}$ afin de déjouer les voies coutumières de l'ascendance et de l'« origine». Comment du théâtre, des rôles et des schémas dramaturgiques établis inventer la forme informe du roman américain? Comment de la mémoire d'un continent déjà saturé de praescriptae mettre le cap vers l'inarchivé? Comment se dépouiller des exégèses et devenir à soi-même son propre Ur-text? Quelques années avant le dénuement de Thoreau au bord de l'étang de Walden et la rhapsodie autochtone de Whitman parmi les herbes folles, Moby Dick répond à ces questions de plusieurs manières. La matière faustienne travaillée par Melville est d'abord redoublée, toujours située à la croisée du drame élisabéthain de Marlowe (The Tragicall History of the Life and Death of Doctor Faustus, 1604) et du Faust I de Goethe (1808), dont l'esthétique médiévale et "rustique » (contrastant avec le classicisme "antique » du Faust II) fait écho aux scènes burlesques que Marlowe emprunte au folklore anglais des Mummers' Plays ${ }^{8}$. Ce double héritage théâtral tendu entre Humanisme et Romantisme est copieusement exhibé par Moby Dick. Il est également soumis à diverses stratégies de contrefaçon : c'est par la reprise littérale de grands thèmes goethéens, l'outrance des références et des attributs méphistophéliques, la prolifération des scènes de pacte et de sabbat, le brouillage du dramatis personae, que Melville affronte avec exubérance «l'impossibilité du roman américain $»^{9}$.

\section{Larguer les amarres}

5 Dès le péritexte de Moby Dick apparaissent deux leitmotive faustiens : l'écœurement provoqué par le trop-plein de culture et l'impuissance du savoir (philologique, théologique, scientifique, technique, littéraire, historiographique) à susciter l'épiphanie de la Baleine originale. Tout comme le Faust goethéen condamne sans appel 
la somme stérile des connaissances engrangées par Wagner, son navrant disciple (« Man läuft euch bei dem ersten Blick davon./Ein Kehrichtfass und eine Rumpelkammer Und höchstens eine Haupt- und Staatsaktion / Mit trefflichen pragmatischen Maximen, / Wie sie den Puppen wohl im Munde ziemen! $\left.»^{10}\right)$, Melville prend d'emblée ses distances avec le pathétique huissier («Sub-Sub Librarian») officiant dans le vestibule surpeuplé de Moby Dick. Il lui attribue l'entière responsabilité de l'archivage (l'« Étymologie » des noms de la Baleine et les quatre-vingt «Extraits») et revêt ironiquement le rôle de simple commentateur.

6 À l'instar du cabinet de travail outrancièrement moyenâgeux que met en scène l'acte I du Faust I ( In einem hochgewölbten, engen gotischen Zimmer » ${ }^{11}$ ), l'avant-texte bibliothécal de Moby Dick répond autant à une exigence d'institutionnalisation (le capital épigraphique rappelle la fidélité d'une tradition qui légitime l'œuvre à venir et oriente sa réception) qu'à une stratégie duplice et déceptive de monstration. Par sa démesure, sa profusion et son hétérogénéité, l'exergue tente bien de domicilier le Léviathan (avant de passer le relais à Ismaël qui échouera lui aussi à circonscrire ce "monstre expansif » au sein d'un savant système), mais il révèle surtout le "mal d'archive $»^{12}$ qui mine de l'intérieur le discours encyclopédique et son vertigineux désir de consignation. Ni les traductions, ni les gloses, ni les variations livresques autour de la Baleine ne suffisent à en saisir la nature et à en quadriller efficacement le corpus au sein d'un Tout harmonieux. Melville renouvelle ainsi la mise en garde goethéenne contre l'ambition de maîtrise et de clôture du savoir (la Sammlung européenne que Pierre-Yves Pétillon oppose à la «fugue » américaine), signalant d'entrée de jeu la faille inhérente à son vain recensement intertextuel: "therefore you must not, in every case at least, take the higgledy-piggledy whale statements, however authentic, in these extracts, for veritable gospel cetology. Far from it. $»^{13}$ Le panorama prétendument exhaustif (" glancing bird's eye view ») sur lequel s'ouvre Moby Dick ne peut donc rester qu'inachevé, à l'état de ruine, telle la Bibliothèque de Faust rongée "de fumée et de moisissure", telle l'« esquisse d'esquisse » à laquelle Ismaël finit par vouer son grand projet taxinomique :

My object here is simply to project the draught of a systemization of Cetology. I am the architect, not the builder. It is a ponderous task; no ordinary letter-sorter in the Post-Office is equal to it [...]. But I now leave my Cetological System standing thus unfinished, even as the great Cathedral of Cologne was left, with the cranes still standing upon the top of the uncompleted tower. For small erections may be finished by their first architects; grand ones, true ones, ever leave the copestone to posterity. God keep me from ever completing anything. This whole book is but a draught-nay, but the draught of a draught. Oh, Time, Strength, Cash, and Patience $^{14}$ !

7 C'est qu'à la tentative inaugurale de somme cétologique ("whole / whale») manque encore l'expérience d'un monde neuf, décapé des anciens récits et lavé de l'usure des mots. Puisque l'Arche du savoir prend l'eau, il faut vite lever l'ancre (" take to the ship ») et changer radicalement de repères («make shift») - quitte à conclure un nouveau pacte avec le diable et à invoquer quelques menaçants Esprits. On verra Achab, le "météorique " Old Thunder, défier le "clair esprit du feu clair» au paroxystique chapitre $\mathrm{CXIX}^{15}$ mais l'invocation fantastique (" there is magic in it " souligne malicieusement Melville) commence dès l'incipit, lorsque l'esprit marin offre à Ismaël la possibilité de s'arracher à l'enclos terrestre de l'habitude et de se délester du passé comme d'un encombrant corps-mort. Ismaël propose ainsi au lecteur de frayer "calmement" sa route à travers les vertes prairies devenues Océan sans limite, à 
l'image goethéenne du «monde paisible» («stille Welt») qu'aperçoit Faust lors de sa promenade aux portes de la ville :

Whenever I find myself growing grim about the mouth; whenever it is a damp, drizzly November in my soul; whenever I find myself involuntarily pausing before coffin warehouses, and bringing up the rear of every funeral I meet ; and especially whenever my hypos get such an upper hand of me, that it requires a strong moral principle to prevent me from deliberately stepping into the street, and methodically knocking people's hats off- then, I account it high time to get to sea as soon as I can. This is my substitute for pistol and ball [...]; I quietly take to the ship $^{16}$.

8 Comme le laisse présager la sombre analogie établie entre le suicide et l'embarquement maritime («This is my substitute for pistol and ball »), Ismaël se prépare également à affronter le risque mortel que comporte toute exposition à la nature sauvage : «I am tormented with an everlasting itch for things remote. I love to sail forbidden seas, and land on barbarous coasts. Not ignoring what is good, I am quick to perceive a horror, and could still be social with it - would they let $m e-»^{17}$. Qu'il soit vagabondage à la lisière du monde, sublime contemplation de l'espace ou troublante "horreur » avec laquelle pactiser, le Démon demeure chez Melville une tentation du Lointain, aiguillée par un violent désir d'autonomie et de connaissance. Il prend ainsi corps avec le tyrannique Achab qui cherche obstinément à "frapper à travers le masque " ("strike though the mask») et à "percer la muraille» («thrusting through the wall») de l'insondable, et se concentre idéalement dans la Blancheur "anomale $»^{18}$ de Moby Dick - cette pure vibration de la couleur (« white living spot ») à laquelle est consacrée le chapitre XLII.

\section{"Dans la légende ou à la foire ": Melville brocanteur de Faust}

9 S'il adopte le mode solaire et aventureux de la fugue dans l'espace, Moby Dick se caractérise également par son esthétique carnavalesque et son décorum faustien, dont les rouages sont démultipliés et soulignés à loisir par Melville. Comment expliquer une telle pléthore de références théâtrales au sein d'une œuvre romanesque qui tend farouchement à s'affranchir de tout ancrage? Comment interpréter l'irrésistible tropisme par lequel l'Europe continue de fournir à l'Amérique le canevas légendaire et les instruments éculés d'un spectacle qui se veut inédit?

De Faust, en effet, Melville ne retient pas seulement la rébellion contre la mélancolie des savoirs et la rigidité des dogmes établis. Il amplifie les scories d'un folklore médiéval qui peuple à divers degrés les œuvres de Marlowe et de Goethe. Bien loin de la posture du philologue, de l'archiviste et de l'historiographe mises à mal par Moby Dick, Melville manie alors la dramaturgie faustienne avec la faconde et l'assurance ostentatoire d'un bateleur roué aux ficelles du mythe. Les formules définitoires du Démon (légion chez Marlowe, intériorisées chez Goethe), resurgissent à l'excès sous sa plume: «Death and damnation, men!» (« Mort et damnation, les gars!»), «Blast it!» ("Que le diable l'emporte !»), «Starbuck now is mine; cannot oppose me now " ${ }^{19}$, "'tis hot as Satan's hoof. It spiralizes in ye; forks out at the serpent-snapping eye $»^{20}$. Cet attirail lourdement symbolique innerve jusqu'à la correspondance. Dans une lettre de juillet 1851, Melville rappelle avec jubilation à Hawthorne la "devise secrète » du roman, énoncée par Achab lorsqu'il baptise son harpon du sang des trois harponneurs païens (Queequeg le Maori, Tashtego l'Indien et Daggoo l'Africain) : «Shall I send you a fin of the 
Whale by way of a specimen mouthful? The tail is not yet cooked - though the hell-fire in which the whole book is broiled might not unreasonably have cooked it all ere this. This is the book's motto (the secret one), - Ego non baptismo in nomine patris, sed in nomine [diabolis] - but make out the rest yourself. ${ }^{21}$

Aux marquages oraux de la subversion diabolique s'ajoute l'incarnation à la fois archétypale et fluctuante du dramatis personae. Tous les masques deviennent réversibles: Achab est à la fois Faust désireux de percer le mystère de la Baleine blanche; mais avec sa cicatrice blafarde, sa jambe d'ivoire, sa boiterie sonore et son harpon, il campe un Méphisto haut en couleurs qui n'a rien à envier à la légende. Son apparition tardive (Melville étire le suspense jusqu'au chapitre XXVIII) est exhaussée par le personnage caricatural de Fedallah. Mystérieusement sorti des tréfonds du Pequod, le «Parsi » forme avec quelques autres adorateurs du feu le doublon maléfique de l'équipage officiel - qui ne s'y trompe pas et identifie en lui le «diable travesti » ( the devil in disguise ») :

Do you believe that cock and bull story about his having been stowed away on board ship? He's the devil, I say. The reason why you don't see his tail, is because he tucks it up out of sight; he carries it coiled away in his pocket, I guess. Blast him! now that I think of it, he's always wanting oakum to stuff into the toes of his boots ${ }^{22}$.

12 Outre l'abondance des attributs, des rôles et des formules-types, on retrouve concentrée aux chapitres XXXVI et XXXVII la structure dramaturgique qui relie depuis Marlowe et Goethe toute scène de tentation à une scène de pacte ( SAVOIR, POUVOIR, VOULOIR... Voilà la triple clé ", résume le Disciple de "Mon Faust »" ${ }^{23}$. Après avoir tenté son équipage au moyen d'une énorme pièce d'or clouée au mât du navire, c'est dans le rhum et le métal qu'Achab scelle l'engagement des marins contre la Baleine blanche. Un sabbat mortifère se substitue alors à la symphonique « Nuit de Walpurgis » du Faust I, ce moment de bascule carnavalesque où la contre-société des bêtes, des sorcières et des feux-follets entraine Faust dans une danse fabuleuse. Chez Melville, le chaos collectif qui signale l'anti-conversion des marins est immédiatement suivi du traditionnel temps de réflexion solitaire, où la part faustienne d'Achab reprend peu à peu conscience ("Gifted with the high perception, I lack the low, enjoying power; damned, most subtly and most malignantly! $\left.{ }^{24}\right)$ mais où la part "sultanesque " du personnage affine également sa stratégie de conquête dans un monologue saisissant de violence. Créature prométhéenne qui se rêve en Tout absolu, Achab dénonce un monde sans transcendance (le "maybe there's naught beyond " lancé à Starbuck répond ainsi à la provocation de Marlowe : «I think hell's a fable »" où ne compte plus que l'affrontement démonique :

Come forth from behind your cotton bags! I have no long gun to reach ye. Come, Ahab's compliments to ye; come and see if ye can swerve me. Swerve me? [...] The path to my fixed purpose is laid with iron rails, whereon my soul is grooved to run. Over unsounded gorges, through the rifled hearts of mountains, under torrents' beds, unerringly I rush! Naught's an obstacle, naught's an angle to the iron way ${ }^{26}$ !

Avec Moby Dick, la mythologie faustienne devenue tall-tale - ce récit mensonger tenant de la farce et de l'exagération qui marque la conquête du grand Ouest américain - se découvre l'actualité d'une "infernale orgie » ou, plus précisément, d'une brocante à ciel ouvert où s'empilent, s'échangent et se démultiplient les signes exutoires du Malin, les costumes de bouc de même que les titres à retraiter, les parchemins à gratter et les pactes à conclure. Chaque support fait ainsi office de palimpseste (contrat d'embarquement des marins, peau tatouée de Queequeg, chair striée de la baleine, front 
ridé d'Achab) ; quant au pacte, il apparaît dès l'incipit et l'invitation faite au lecteur à appareiller vers la haute mer («Call me Ishmaël»). Melville décline ensuite ce leitmotiv sous toutes ses formes : cela va de la signature de Queequeg (chapitre XVIII) au marché passé entre Achab et Fedallah au chapitre LXXIII, de la tentation du doublon proposé aux marins en échange de la capture de Moby Dick (chapitre XXXVI) à la forge infernale des armes (chapitre CXIII), en passant par le raffermissement du serment « de violence et de vengeance » d'Ismaël au chapitre XLI et la rédaction de son testament au chapitre XLIX : "finally considering in what a devil's chase I was implicated, touching the White Whale: taking all things together, I say, I thought I might as well go below and make a rough draft of my will. $\aleph^{27}$ Ismaël apparait ainsi comme le plus fidèle avatar de Faust, manquant de succomber à la danse macabre imposée par Achab mais demeurant, après le naufrage du Pequod, le seul maître de l'écriture.

Que retenir de ce mouvant carnaval orchestré par Moby Dick et dans quelle mesure participe-t-il à la liquidation de l'héritage européen ? On l'a vu, Melville commence par rassembler les fragments d'une Bibliothèque-source, mais c'est pour mieux la faire fuir et inventer par d'autres moyens la geste foraine et luxuriante de l'Amérique. Seul l'arrachement au «maudit cachot » liminaire libère en effet la possibilité d'éprouver pour soi-même la "vivante Nature $»^{28}$ évoquée par Faust et d'exposer son œil à l'éblouissante beauté de la "Wilderness ». Condamné d'avance, le misérable «adjointde-l'adjoint » ne survit pas au seuil empoussiéré de Moby Dick. Comme chez Marlowe et Goethe, le voyage sans carte ni boussole auquel nous convie Melville, déjà entamé avec l'utopique Mardi (1849), a bien sûr un prix : l'exil volontaire hors de l'espace livresque permet certes à Ismaël de faire l'expérience d'Autrui, mais il se paye aussi de l'aliénation, de l'égarement, de la fragmentation intime et de l'apocalypse finale.

Tout au long de ce trajet paradigmatique qui nous mène de l'obscurité vermoulue des registres jusqu'au "still point» de l'ultra-blanc, Melville entrelarde son récit de clinquantes références faustiennes, dont il importe de souligner la fonction déictique et réflexive. Elles jouent en effet le même rôle de panneaux indicateurs que les topoï gothiques exploités par Julien Gracq. On se souvient de l'étonnant avis adressé au lecteur d'Au Château d'Argol: "Quant aux machines de guerre qui dans ce récit sont mises en œuvre çà et là, et destinées à faire mouvoir les ressorts toujours malaisément maniables de la terreur, un soin particulier a été apporté à ce qu'elles ne fussent, et surtout ne parussent pas inédites, et pussent par conséquent jouer du plus loin possible le rôle d'un signal avertisseur. ${ }^{29}$ Roman grandiloquent de la perversion et de la contrefaçon, Moby Dick revêt ainsi la forme d'un théâtre de machines lancé à plein régime, dévoilant au lecteur-spectateur les coulisses et les chaudières où l'auteur enfourne les matériaux de la fabrique romanesque. L'apparente naïveté (voire maladresse) de la reprise littérale cache ici une extraordinaire maîtrise dans le traitement des coordonnées faustiennes et dans la connaissance des «emplois » qui seront ensuite repris par Valéry et Pessoa en vue d'un Faust III $^{30}$.

16 C'est par son esthétique de pacotille que Melville emblématise les actes d'un romancontinent à la fois épique, comique et tragique. Ainsi «cueilli à l'état de fantoche $»^{31}$, l'héritage faustien de Moby Dick n'est plus un poids qui menace le vivant; exacerbé à l'extrême, disséminé dans l'épaisseur foisonnante du texte-Léviathan, le legs se fait 
léger masque de carton, persona de papier à travers lequel résonne la voix inouïe de l'«auctor ». Derrière le Faust-legend et la parade de cirque, l'héritage redevient ce mince, fragile et réversible vélin qui permet à Melville de s'affranchir de l'emprise du passé et de signer pour la postérité.

\section{NOTES}

1. La formule a été introduite en 1941 par le critique Francis Otto Matthieussen (American Renaissance: Art and Expression in the Age of Emerson and Whitman, Kessinger Publishing, LLC, 2007). Elle désigne une sorte d'âge d'or de la littérature américaine qui s'étend des années 1830 jusqu'à la fin de la Guerre civile (1865). La demi-décennie 1850-1855, marquée par la publication des grands textes d'Hawthorne (The Scarlet Letter et The House of the Seven Gables, 1950-1951), de Melville (Moby-Dick, 1851), de Thoreau (Walden, 1954) et de Whitman (Leaves of Grass, 1955) est considérée comme l'acmé de ce mouvement fondateur par lequel les écrivains américains s'affranchissent de leur dépendance coloniale vis-à-vis de la littérature britannique. Pour une mise en perspective contemporaine du concept de Renaissance américaine et de ses enjeux, voir David Reynolds, Beneath the American Renaissance: The Subversive Imagination in the Age of Emerson and Melville, Harvard University Press, 1989.

2. Goethe, Faust, trad. J. Amsler revue par O. Mannoni, Paris, Gallimard, « Folio bilingue », 2007, p. 60.

3. Herman Melville, Hawthorne et ses mousses, trad. P. Leyris, Paris, Gallimard, 1986, p. 233.

4. Ralph Waldo Emerson, Nature, dans The Spiritual Emerson: Essential Writings, éd. David M. Robinson, Boston, Beacon Press, 2003, p. 23. (« Notre âge est rétrospectif. Il bâtit les mausolées de nos pères. Il écrit des biographies, des histoires et de la critique. Les générations précédentes regardaient Dieu et la nature en face ; nous le faisons à travers leurs yeux. Pourquoi ne pas jouir, nous aussi, d'une relation originale à l'univers? 侮...骨 pourquoi devrions-nous alors tâtonner parmi les os secs du passé ou faire de la génération vivante une mascarade à la garde-robe surannée? Le soleil brille aujourd'hui aussi.» Essais, trad. A. Wicke, Paris, Michel Houdiard Éditeur, 2010, p. 15)

5. Pierre-Yves Pétillon, La Grand-route, Paris, Seuil, 1979.

6. "Il ne te suffit pas d'être toi-même un livre ?... », Paul Valéry, "Mon Faust », Paris, Gallimard, 1946, p. 37.

7. Michel Foucault, « Nietzsche, la généalogie, l'histoire », Dits et écrits II, Paris, Gallimard, 1994, p. 143.

8. Comme le rappelle François Laroque, il s'agit de saynètes populaires que l'on jouait à Noël et à Pâques, dont les protagonistes se paraient de masques d'animaux et qui traitaient de thèmes carnavalesques (luxure, goinfrerie, démembrement, danses macabres). Cf. Christopher Marlowe, Le Docteur Faust, trad. F. Laroque et J.-P. Villquin, Paris, Flammarion, «GF bilingue », 1997, p. 22-25.

9. Jacques Cabau, La Prairie perdue, Paris, Seuil, 1981, p. 12.

10. "Rien qu'à vous voir, on se sauve : / une tonne à déchets, et un bric-à-brac, / au mieux une tragédie classique / frappée de valables maximes pragmatiques, / telles qu'elles conviennent dites par des pantins !» Goethe, op. cit., p. 74-75.

11. Ibid., p. 56. 
12. Jacques Derrida, Mal d'archive, Paris, Galilée, 1995.

13. Herman Melville, Moby Dick, London, Penguin Books Popular Classics, 1994, p. 10. («Aussi ne doit-on pas toujours prendre ce salmigondis de citations, si authentiques soient-elles, pour un véritable évangile de cétologie. Loin s'en faut. » Moby-Dick, Euvres III, trad. P. Jaworski et P. Leyris, Paris, Gallimard, «Bibliothèque de la Pléiade », 2006, p. 5)

14. Melville, op. cit., p. 139 et 149. («Mon seul objet est d'esquisser le projet de taxinomie cétologique. Je suis l'architecte, non le maçon. Mais c'est là une tâche considérable; aucun vulgaire trieur de lettres du service des postes ne saurait y suffire [...]. Je laisse mon système cétologique inachevé, comme est demeurée inachevée la grande cathédrale de Cologne, avec sa grue oisive au sommet de la tour incomplète. Car si les petits édifices peuvent être terminés par leur architecte primitif, les grands, les vrais, confient toujours la pose du couronnement à la postérité. Dieu me garde de jamais rien parfaire. Ce livre tout entier n'est qu'une esquisse... même pas : l'esquisse d'une esquisse. Ô Temps, Force, Argent, Patience !» Trad. p. 159 et 170)

15. Voir les analyses de Peter Szendy dans Les Prophéties du texte-Léviathan (Paris, Minuit, 2004, p. 97-101).

16. Melville, op. cit., p. 21. ("Quand je sens l'amertume plisser mes lèvres, quand bruine dans mon âme un humide novembre et que je me surprends à faire halte, malgré moi, devant les marchands de cercueils, à me glisser dans le premier cortège funèbre que je croise, et, surtout, quand la noire mélancolie me tient si fort que seul un robuste sens moral peut m'empêcher de descendre d'un pas décidé dans la rue et d'envoyer méthodiquement valser les chapeaux des passants alors, j'estime nécessaire de m'embarquer sans délai. D'autres auraient recours à un pistolet chargé [...] ; moi, tranquillement je lève l'ancre. » Trad. p. 21)

17. Ibid., p. 26. ( «j'ai, des choses lointaines, une inguérissable démangeaison. J'aime immodérément sillonner les mers interdites et aborder aux rivages barbares. Sans méconnaître ce qui est bon et bien, je suis prompt à percevoir une horreur et puis même établir avec elle, si nul n'y fait obstacle, la plus cordiale relation. » Trad. p. 26-27)

18. «L'Anomal est toujours à la frontière, sur la bordure d'une bande ou d'une multiplicité ; il en fait partie, mais la fait déjà passer dans une autre multiplicité, il la fait devenir, il trace une ligneentre. C'est aussi l'“outsider" : Moby Dick, ou bien la Chose, l'Entité de Lovecraft, terreur. » Gilles Deleuze, Claire Parnet, Dialogues, Paris, Flammarion, 1996, p. 54.

19. Melville, op. cit., p. 168. («Starbuck est à moi, désormais il ne peut s'opposer. » Trad. p. 193) La formule péremptoire d'Achab rappelle sans détour le blasphématoire « Consummatum est. The bill is ended. / And Faustus hath bequeathed his soul to Lucifer » de l'acte II du Doctor Faustus, par lequel Marlowe détourne les dernières paroles du Christ sur la croix. («Tout est accompli. Cet acte est conclu, / Et Faust a légué son âme à Lucifer. », Christopher Marlowe, op. cit., p. 94-95)

20. Ibid. («C'est brûlant comme le sabot de Satan. Ce rhum descend en vous comme une vrille, vous met dans le regard la hargne venimeuse du serpent... »)

21. The Letters of Herman Melville, ed. Merrell R. Davis et William H. Gilman, New Haven, Yale University Press, 1960, p. 133. («Vous enverrai-je une nageoire de la Baleine pour y goûter? La queue n'est pas encore bien cuite, bien que le feu d'enfer où flambe tout le livre ait déjà pu, raisonnablement faire son œuvre. Voici la devise du livre (la secrète), - Ego non baptismo in nomine patris, sed in nomine - mais je vous laisse achever le reste.» Melville, D'où viens-tu, Hawthorne? trad. P. Leyris, Paris, Gallimard, 1986, p. 126-127)

22. Melville, op.cit., p.315. («Croyez-vous vraiment à cette histoire abracadabrante d'embarquement clandestin? C'est le diable, vous dis-je. La raison pour laquelle on ne voit point sa queue, c'est qu'il la dissimule aux regards, lovée dans sa poche, j'imagine. Maudit soit-il! Et maintenant que j'y pense, il demande toujours de l'étoupe, dont il bourre la pointe de ses bottes. " Trad. p. 362)

23. Paul Valéry, op. cit., p. 129. 
24. Melville, op. cit., p. 170. (" Doué de la plus haute perception, je suis privé de l'humble faculté de jouissance. » Trad. p. 195)

25. « Moi je pense que l'Enfer n'est qu'une fable », Christopher Marlowe, op. cit., p. 98-99.

26. Melville, op. cit., p. 171. («Sortez de derrière vos balles de coton! Je n'ai pas de fusil dans les mains. Venez, Achab vous présente ses compliments; approchez et voyez si vous pouvez me dérouter. Me dérouter [...] ? La voie qui mène au but que je me suis fixé est un rail d'acier auquel sont ajustés les roués de mon âme. Infailliblement je me rue, franchis les ravins insondés, traverse le cœur des montagnes, file sous le lit des torrents! Nul coude, nul obstacle sur mon chemin de fer! » Trad. p. 196)

27. Ibid., p. 227 (« considérant enfin que, poursuivant le cachalot blanc, j'étais engagé dans une chasse de tous les diables - je me dis que tout compte fait, j'avais intérêt à descendre au poste pour y rédiger le brouillon de mes dernières volontés. » Trad. p. 259)

28. Goethe, op. cit., p. 60-61.

29. Julien Gracq, « Avis au lecteur », Au Château d'Argol, Paris, José Corti, 1938, p. 10.

30. Voir à ce sujet Julia Peslier, La Pensée à l'œuvre. Chantiers de Faust ouverts par Goethe (Valéry, Pessoa, Mann, Boulgakov), Thèse de doctorat dirigée par T. Samoyault et J.-M. Rey, Université de Paris 8, 2007.

31. Paul Valéry, op. cit., p. 7.

INDEX

oeuvrecitee Moby Dick, Faust

Mots-clés : roman-monstre

Keywords : novel

Palabras claves : novela

Palavras-chave : novela

\section{AUTEURS}

\section{ANNE BOURSE}

Docteure en littérature comparée

Centre parisien d'études critiques 